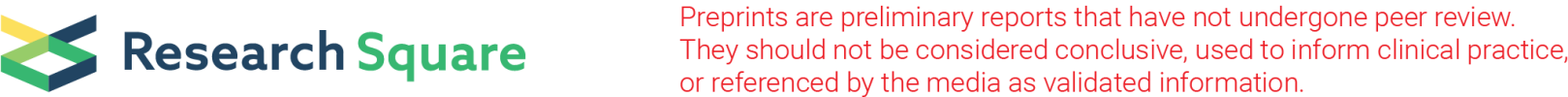

\section{The impact of the COVID-19 pandemic on the emergency department visits: a retrospective analysis in Shanghai, China}

Wei Long ( $\square$ longwei2290@163.com )

Shanghai Sixth People's Hospital https://orcid.org/0000-0002-6832-7638

Jiudong Hu

Shanghai Sixth Peoples Hospital

Lijuan Li

Shanghai Sixth Peoples Hospital

Sheng Zuo

Shanghai Sixth Peoples Hospital

Qian Yang

Shanghai Sixth Peoples Hospital

Zenghua Ren

Shanghai Sixth Peoples Hospital

Research article

Keywords: COVID-19, Emergency Department, Pandemic

Posted Date: August 29th, 2020

DOI: https://doi.org/10.21203/rs.3.rs-49525/v2

License: () (1) This work is licensed under a Creative Commons Attribution 4.0 International License.

Read Full License 


\section{Abstract}

Background The novel coronavirus diseases (COVID-19) has led to a pandemic and affected people's lives greatly, including their health seeking behavior. We aimed to evaluate the impact of the current COVID-19 pandemic on characteristics and trends of emergency department (ED) visits in Shanghai, China.

Methods This was a retrospective observational study using medical record databases from the Shanghai Sixth People's Hospital (East Campus) for years 2016 through 2020. All the patients referred to the ED between January 2016 and June 2020 were retrospectively reviewed. January 1, 2020, was chosen as the cutoff date for the statistical analysis and data of January and February in 2020 was compared with the same period of 2019.

Results During the first two months of 2020, there was a $60.9 \%$ decline in ED visits when compared with the same period of 2019 (9,904 vs. 25,316, respectively), and the waiting time in ED has been greatly reduced correspondingly (12 \pm 4 vs. $66 \pm 19 \mathrm{~min}, p<0.001)$; ED visits for acute ischemic stroke (AIS) and acute coronary syndrome(ACS) decreased by $53.9 \%$ and $41.2 \%$ respectively; proportion of intravenous thrombolysis for AIS has dropped( $42.1 \%$ vs. $11.4 \%, p=0.003)$, and percutaneous coronary intervention for ACS was similar (70.6\% vs. $63.3 \%, p=0.668)$; and onset-to-door time (ODT) of these patients increased significantly (AIS: 217(136-374) vs. 378(260-510)min, ACS: 135(85-195) vs. 226(155-368)min, all $p<0.001)$.

Conclusion The outbreak of COVID-19 pandemic was correlated with a significant decline in the number of ED visits including AIS and ACS patients when compared to the pre-COVID-19 period. ODT of AIS and ACS patients increased significantly. Raising public awareness is necessary to avoid serious healthcare and economic consequences of undiagnosed and untreated stroke and myocardial infarction attack.

\section{Background}

The novel coronavirus diseases (COVID-19), began in Wuhan, China since December 2019, has led to a pandemic, accounting for more than 15.8 million worldwide infections and more than 630,000 death (as of July 25,2020$)[1,2]$. After the outbreak of COVID-19, the Chinese government has taken unprecedented measures to stem further dissemination of the virus. These include isolating ill persons, contact tracing, quarantine of exposed persons, travel restrictions, school and workplace closures, cancellation of mass gatherings, and hand washing, among others. The Chinese government shut down crowded public places such as shopping malls, restaurants, cinemas, cancelled or postponed large public events, encouraged people to stay at home as much as possible, and venture out only to get food or medical help. These strategies appear to be effective and no new domestic cases were confirmed on the Chinese mainland by March $18[3,4]$. 
The pandemic has affected people's lives greatly, including their health seeking behavior. However, the impact of the pandemic on emergency care has not been reported. In the present investigation, we aimed to evaluate the impact of the current COVID-19 pandemic on characteristics and trends of emergency department (ED) visits including critical diseases such as acute ischemic stroke (AIS) and acute coronary syndrome (ACS) in Shanghai, China.

\section{Methods}

This was a retrospective observational study using medical record databases from the Shanghai Sixth People's Hospital (East Campus) for years 2016 through 2020. The study hospital is a tertiary hospital and regional medical center of southeast Shanghai, China, centre with catchment area exceeding 300,000 inhabitants during the study period. The study was approved by the local ethics committee. Written informed consent was waived due to the retrospective character of the investigation.

All patients referred to the ED between January 2016 and June 2020 were retrospectively reviewed. In the study ED, all patients visit the same ED regardless of their indication for emergency visit and all ED physicians have similar options available to examine patients, i.e. the same radiological services and laboratory examinations are available to all physicians and for all patients. Specialists (i.e. internists, surgeons, neurologists and emergency physicians), residents and other acute care physicians work alongside each other.

Principal conditions are based on the first-listed diagnosis on the ED visit record and are determined by the International Classification of Diseases, Tenth Revision, Clinical Modification [ICD-10-CM].

Post-COVID era was defined as after January 1,2020 , as it was the starting date for social restrictions, and the COVID-19 pandemic began to impact public life in China. January and February 2020 were the peak of the pandemic in China, and no new domestic cases were confirmed on the Chinese mainland by March 18, so we compared the data of January and February in 2020 with the same period of 2019.

Discrete variables are expressed as percentage and continuous variables as mean \pm standard deviation (SD) for variables normally distributed and as median with interquartile range (IQR) for not normally distributed data. Comparability of groups was analyzed by $\chi^{2}$ test, two-sampled $t$ test, Mann-Whitney $U$ test, as appropriate; $p<0.05$ was defined as being statistically significant. Statistical analysis was performed using IBM SPSS Statistics Version 20.

\section{Result}

\section{Trends in ED visits between 2016 and 2020}

There has been a steadily $17.5-28.4 \%$ annual increase in ED visits over the past four years from January 2016 to December 2019, and the annual total ED visits increased 83.0\% from 89,340 in 2016 to 163,496 in 2019 (Fig. 1, Fig. 2). But ED visits declined significantly after onset of COVID-19 from January 2020. 
There was a $60.9 \%$ decline in ED visits during the first two months of 2020 when compared with the same period of 2019 (9,904 versus 25,316, respectively). After March 18, the COVID-19 pandemic was nearing its end in China, ED visits began to increase again (Fig. 2).

\section{Characteristics Of Visits Before And After The Covid-19}

In January-February 2019, the top five common conditions for visits in ED were acute bronchitis, acute pharyngitis, sprains and strains, superficial injury and contusion, and abdominal pain. After onset of COVID-19 from January 2020, the top five became abdominal pain, pneumonia, dizziness and vertigo, sprains and strains, and superficial injury and contusion (Table 1).

Table 1

The 5 most common conditions seen in the ED before and after the COVID-19

\begin{tabular}{|lllll|}
\hline Principal condition & \multicolumn{2}{c}{ January-February 2019 } & \multicolumn{2}{c|}{ January-February 2020 } \\
\cline { 2 - 4 } & Number & Rank & Number & Rank \\
\hline Acute bronchitis & 4,556 & 1 & 715 & \\
\hline Acute pharyngitis & 3,797 & 2 & 646 & \\
\hline Sprains and strains & 3,037 & 3 & 851 & 4 \\
\hline Superficial injury and contusion & 2,784 & 4 & 782 & 5 \\
\hline Abdominal pain & 2,531 & 5 & 1,485 & 1 \\
\hline Pneumonia & 1782 & & 1,088 & 2 \\
\hline Dizziness and vertigo & 1253 & & 922 & 3 \\
\hline
\end{tabular}

After onset of COVID-19, the waiting time in ED has been greatly reduced during the first two months of 2020 when compared with the same period of 2019 (12 \pm 4 vs. $66 \pm 19 \mathrm{~min}, p<0.001)$.

\section{Characteristics of AIS and ACS patients before and after the COVID-19 in ED}

The overall number of AIS and ACS patients admitted to our ED during the first two months of 2020 decreased $53.9 \%$ and $41.2 \%$ respectively after onset of COVID-19 when comparing with the same period of 2019 (AIS: 76 vs. 35, ACS: 51 vs. 30, Table 2).

Proportion of intravenous thrombolysis (IVT) for AIS has dropped ( $42.1 \%$ vs. $11.4 \%, p=0.003$ ), and percutaneous coronary intervention (PCl) for ACS was similar (70.6\% vs. $63.3 \%, p=0.668)$.

Onset-to-door time (ODT) of these patients increased significantly during the first two months of 2020 when comparing with the same period of 2019 (AIS patients: 217(136-374) vs. 378(260-510) min, ACS patients: $135(85-195)$ vs. $226(155-368) \mathrm{min}$, all $p<0.001$, Table 2$)$. 
Table 2

Characteristics of AIS and ACS patients in ED before and after the COVID-19

\section{Characteristics}

January-February 2019

January-February 2020

p-value

AIS

Number of patients

76

35

Age (y), mean (SD)

$74 \pm 13$

$72 \pm 12$

0.442

Male sex, n (\%)

37(48.7)

19(54.3)

0.731

ODT (min), median (IQR)

217(136-374)

$378(260-510)$

$<0.001$

IVT, n (\%)

32(42.1)

4(11.4)

0.003

ACS

Number of patients

51

30

Age (y), mean (SD)

$62 \pm 11$

$61 \pm 12$

0.703

Male sex, n (\%)

34(66.7)

19(63.3)

0.950

ODT (min), median (IQR)

135(85-195)

226(155-368)

$<0.001$

$\mathrm{PCl}, \mathrm{n}(\%)$

36(70.6)

19(63.3)

0.668

Data are presented as number (\%), median (IQR) or mean \pm SD.

Abbreviations: AIS, acute ischemic stroke; ODT: onset-to-door time; IVT, intravenous thrombolysis:

ACS, acute coronary syndrome; $\mathrm{PCl}$, percutaneous coronary intervention.

\section{Discussion}

ED serves as an important source of public healthcare system for many patients, and they can seek care at any ED, regardless of their ability to pay, and this care is available 24 hours a day. In addition, because of the growth of emergency medicine as a physician specialty, patients see the ED as a source of highquality care. In many countries the number of ED visits is growing faster than the population [5-7].

In our study, the number of ED visits increased significantly by $17.5-28.4 \%$ a year over the past four years, but the trend has been interrupted by the COVID-19 pandemic. In this single-center, retrospective observational study, we report a significant decline (60.9\%) in the number of ED visits after outbreak of the COVID-19 pandemic when compared to the pre-COVID period.

ED overcrowding is a serious issue worldwide $[8,9]$. This occurs $12-73 \%$ of the time according to a nationwide study in the United States [10]. ED overcrowding was attributed to inappropriate use of the ED by a large volume of non-urgent patients. In survey studies, ED overcrowding has been reported to cause delays in diagnosis, delays in treatment, decreased quality of care, and poor patient outcomes. ED 
overcrowding could potentially affect anyone who suffers unexpected severe illness or injury requiring time sensitive emergency treatment.

China has a large population, and ED overcrowding is widespread in many big cities and has reportedly reached crisis proportions $[11,12]$. In China, the most common conditions seen in the ED were acute bronchitis, acute pharyngitis, Sprains and strains, superficial injury and contusion, which are usually not so urgent and don't need emergency-based services.

After the outbreak of COVID-19, the Chinese Government had taken some strict measures to prevent people from gathering or moving unnecessarily. People are required to avoid public places, and wear a mask when going out. These measures have played a key role in cutting off the transmission route of COVID-19, and the most frequent conditions seen in the ED mentioned above have been reduced at the same time. Along with reduction of numbers of the non-urgent patients, ED overcrowding has been relieved. As seen in the study, the waiting time in ED has been greatly reduced. In terms of alleviating ED overcrowding, the pandemic outbreak is not a bad thing, as limited ED resource can be allocated to patients who need emergency care really.

We additionally observed a reduction in the overall number of AIS and ACS patients admitted to our ED and a significant increase in ODT of these patients when comparing the current lockdown period with the same period of 2019 in our study. Reasons for this phenomenon are not understood although some suggest that patients' fear of exposure to COVID-19 could have contributed to their wait-and-see behavior. The fear of infection and adhering to advice from public health organizations and government officials likely outweighs the concern over mild cardio-cerebrovascular symptoms. Milder symptoms of an acute cardio-cerebrovascular event, which could have historically prompted patients to seek medical attention, receive acute treatment and aggressive secondary prevention, may now be minimized to the point that these patients will stay at home and not present to the emergency room.

Irrespective of the causes, these phenomenon are worrisome, because we all know that AIS and ACS are time dependent diseases, IVT in ischemic stroke and PCI in myocardial infarction are extremely timesensitive [13-15]. Thrombolysis started within the first 60 minutes after onset is associated with best outcomes for patients with AIS, and over the entire 4.5-hour guideline-endorsed tissue plasminogen activator time window, rates of being disability-free at discharge and discharge to home decay more rapidly in the first 100 to 170 minutes of stroke onset to treatment time, whereas independent ambulation at discharge and in-hospital mortality decline in a linear fashion throughout [16-18]. Short onset to balloon time was associated with lower in-hospital mortality and better long term clinical outcome in patients with myocardial infarction having PCl. Furthermore, a symptom-onset-to-balloon time $>4 \mathrm{~h}$ was identified as independent predictor of one-year mortality $[19,20]$.

We are concerned that the delay in seeking medical care might be accompanied by a substantial increase in post-acute complications and long-term disability even mortality due to the lack of appropriate acute management and implementation of secondary prevention interventions. 
To our knowledge, this is the first study to publish data about the impact of the current COVID-19 pandemic on characteristics and trends of ED visits in China, and our study verifies observations recently made by researchers in different countries [21-24].

However, some limitations of the present study should be recognized. The main limitation of this study is the relatively small sample size as a single-center experience. Taking into account the limited population size in the study, multi-center studies enrolling a larger number of patients is desirable to validate our data. Second, there are many critical diseases in ED, but only two of them were chosen in this study for analysis. We think AIS and ACS are the most typical time dependent diseases in ED, and it's enough to discover the delayed ODT phenomenon through them.

\section{Conclusion}

These data reveal that the COVID-19 pandemic had a strong influence on ED visits in China. The number of ED visits including AIS and ACS patients reduced greatly, and ODT of AIS and ACS patients increased significantly. Health administration department should raise and sustain public awareness for the critical disease such as stroke and myocardial infarction, in particular regarding the importance of timely diagnosis and treatment of acute symptoms, and patients should be encouraged to seek medical care and be assured that hospitals make every effort to prevent contact to potentially infectious patients. All of this is paramount to avoid serious healthcare and economic consequences resulting from undiagnosed and untreated critical disease.

\section{Abbreviations}

ED: Emergency Department

SD: Standard Deviation

IQR: interquartile range

AIS: acute ischemic stroke

ACS: acute coronary syndrome

IVT: intravenous thrombolysis

PCl: percutaneous coronary intervention

ODT: onset-to-door time

\section{Declarations}

\section{Availability of data and materials}


The datasets analyzed during the current study are available from the corresponding author on reasonable request.

\section{Acknowledgements}

Not applicable.

\section{Funding}

There was no funding for this study.

\section{Author information}

\section{Affiliations}

Department of Emergency and Geriatrics, Shanghai Sixth People's Hospital East Affiliated to Shanghai University of Medicine \& Health Sciences, 222 Huan Hu Xi San Road, Shanghai, 201306, China

\section{Contributions}

WL had full access to all the data in the study and take responsibility for the integrity of the data and the accuracy of the data analysis. WL and LL conceived the idea and supervised the study. WL and JH designed the study. SZ and ZR acquired the data. QY and ZR analyzed and interpreted data. WL and QY drafted the manuscript. All authors have read and approved this manuscript and submission.

Corresponding author

Correspondence to Wei Long

\section{Ethics declarations}

Ethics approval and consent to participate

The study was approved by the Ethics and Research Committee of the Shanghai Sixth People's Hospital (East Campus). The institutional Ethics and Research Committee waived the requirement for consent because the project is a retrospective analysis of routinely collected administrative data.

\section{Consent for publication}

Not applicable.

\section{Competing interests}

The authors declare that they have no competing interests.

\section{References}


1. Zhu N, Zhang D, Wang W, Li X, Yang B, Song J, et al. A Novel Coronavirus from Patients with Pneumonia in China, 2019. N Engl J Med. 2020;382:727-33.

2. Guan WJ, Ni ZY, Hu Y, Liang WH, Ou CQ, He JX, et al. Clinical Characteristics of Coronavirus Disease 2019 in China. N Engl J Med. 2020;382:1708-20.

3. Leung K, Wu JT, Liu D, Leung GM. First-wave COVID-19 transmissibility and severity in China outside Hubei after control measures, and second-wave scenario planning: a modelling impact assessment. Lancet. 2020;395:1382-93.

4. Chowdhury R, Luhar S, Khan N, Choudhury SR, Matin I, Franco OH. Long-term strategies to control COVID-19 in low and middle-income countries: an options overview of community-based, nonpharmacological interventions. Eur J Epidemiol. 2020;13:1-6.

5. Hooker EA, Mallow PJ, Oglesby MM. Characteristics and Trends of Emergency Department Visits in the United States (2010-2014). J Emerg Med. 2019;56:344-51.

6. Shaha M, Gmur S, Schoenenberger AW, Gerber FS, Exadaktylos AK. Trends and characteristics of attendance at the emergency department of a Swiss university hospital: 2002-2012. Swiss Med Wkly. 2015;145:w14141.

7. Tang N, Stein J, Hsia RY, Maselli JH, Gonzales R. Trends and characteristics of US emergency department visits, 1997-2007. JAMA. 2010;304:664-70.

8. Di Somma S, Paladino L, Vaughan L, Lalle I, Magrini L, Magnanti M. Overcrowding in emergency department: an international issue. Intern Emerg Med. 2015;10:171-5.

9. Wretborn J, Henricson J, Ekelund U, Wilhelms DB. Prevalence of crowding, boarding and staffing levels in Swedish emergency departments - a National Cross Sectional Study. BMC Emerg Med. 2020;20:50.

10. Trzeciak S, Rivers EP. Emergency department overcrowding in the United States: an emerging threat to patient safety and public health. Emerg Med J. 2003;20:402-5.

11. Wang Z, Xiong X, Wang S, Yan J, Springer M, Dellinger RP. Causes of Emergency Department Overcrowding and Blockage of Access to Critical Services in Beijing: A 2-Year Study. J Emerg Med. 2018;54:665-73.

12. Chan SS, Cheung NK, Graham CA, Rainer TH. Strategies and solutions to alleviate access block and overcrowding in emergency departments. Hong Kong Med J. 2015;21:345-52.

13. Meretoja A, Keshtkaran M, Saver JL, Tatlisumak T, Parsons MW, Kaste M, et al. Stroke thrombolysis: save a minute, save a day. Stroke. 2014;45:1053-8.

14. Shiomi H, Nakagawa Y, Morimoto T, Furukawa Y, Nakano A, Shirai S, et al. Association of onset to balloon and door to balloon time with long term clinical outcome in patients with ST elevation acute myocardial infarction having primary percutaneous coronary intervention: observational study. BMJ. 2012;344:e3257.

15. Mulder M, Jansen IGH, Goldhoorn RB, Venema E, Chalos V, Compagne KCJ, et al. Time to Endovascular Treatment and Outcome in Acute Ischemic Stroke: MR CLEAN Registry Results. Circulation. 2018;138:232-40. 
16. Kim JT, Fonarow GC, Smith EE, Reeves MJ, Navalkele DD, Grotta JC, et al. Treatment With Tissue Plasminogen Activator in the Golden Hour and the Shape of the 4.5-Hour Time-Benefit Curve in the National United States Get With The Guidelines-Stroke Population. Circulation. 2017;135:128-39.

17. Saver JL, Fonarow GC, Smith EE, Reeves MJ, Grau-Sepulveda MV, Pan W, et al. Time to treatment with intravenous tissue plasminogen activator and outcome from acute ischemic stroke. JAMA. 2013;309:2480-8.

18. Jahan R, Saver JL, Schwamm LH, Fonarow GC, Liang L, Matsouaka RA, et al. Association Between Time to Treatment With Endovascular Reperfusion Therapy and Outcomes in Patients With Acute Ischemic Stroke Treated in Clinical Practice. JAMA. 2019;322:252-63.

19. De Luca G, Suryapranata H, Zijlstra F, van 't Hof AW, Hoorntje JC, Gosselink AT, et al. Symptom-onsetto-balloon time and mortality in patients with acute myocardial infarction treated by primary angioplasty. J Am Coll Cardiol. 2003;42:991-7.

20. Cannon CP, Gibson CM, Lambrew CT, Shoultz DA, Levy D, French WJ, et al. Relationship of symptomonset-to-balloon time and door-to-balloon time with mortality in patients undergoing angioplasty for acute myocardial infarction. JAMA. 2000;283:2941-7.

21. Siegler JE, Heslin ME, Thau L, Smith A, Jovin TG. Falling stroke rates during COVID-19 pandemic at a comprehensive stroke center. J Stroke Cerebrovasc Dis. 2020;29:104953.

22. Hoyer C, Ebert A, Huttner HB, Puetz V, Kallmunzer B, Barlinn K, et al. Acute Stroke in Times of the COVID-19 Pandemic: A Multicenter Study. Stroke. 2020;51:2224-7.

23. Metzler B, Siostrzonek P, Binder RK, Bauer A, Reinstadler SJ. Decline of acute coronary syndrome admissions in Austria since the outbreak of COVID-19: the pandemic response causes cardiac collateral damage. Eur Heart J. 2020;41:1852-3.

24. Braiteh N, Rehman WU, Alom M, Skovira V, Breiteh N, Rehman I, et al. Decrease in acute coronary syndrome presentations during the COVID-19 pandemic in upstate New York. Am Heart J. 2020;226:147-51.

\section{Figures}




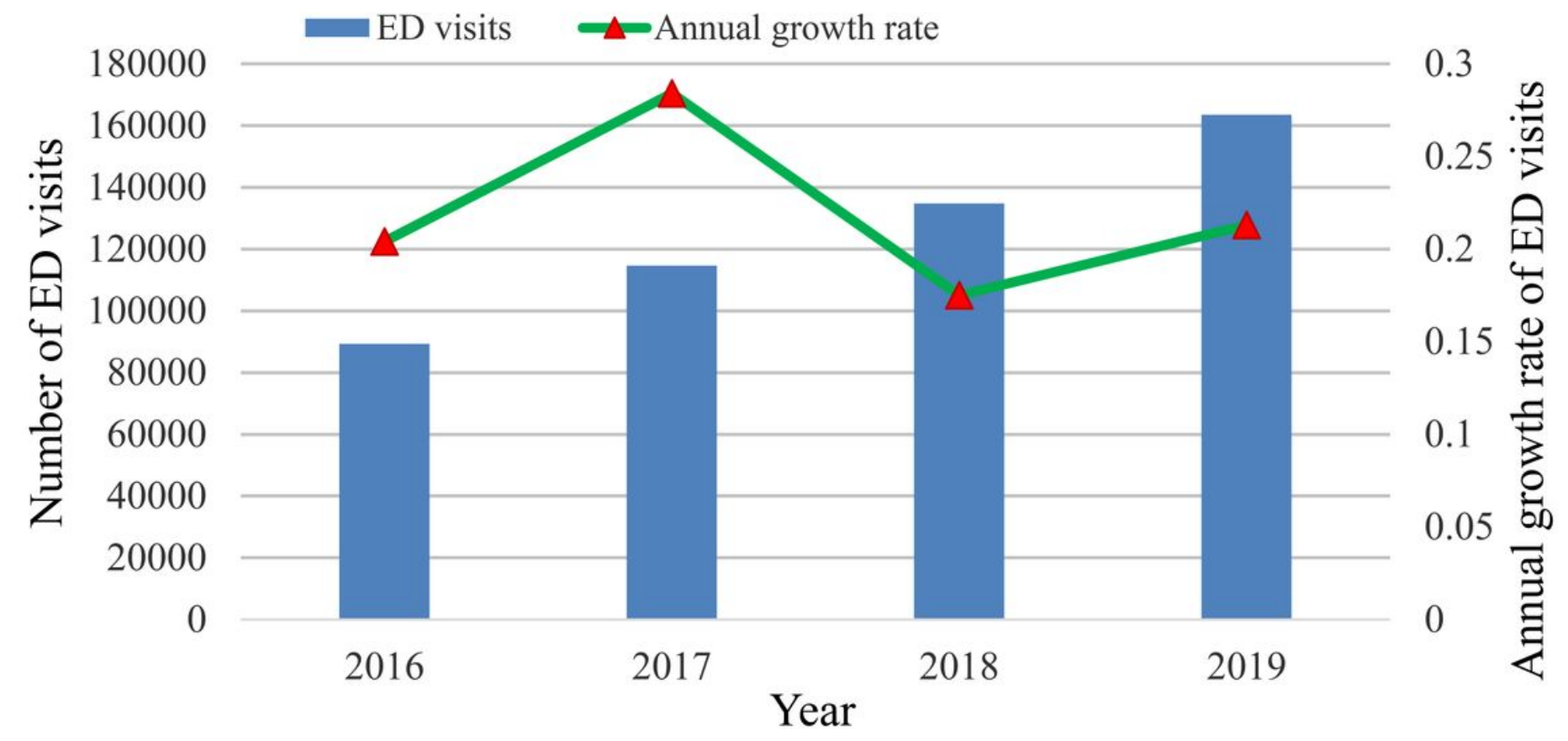

Figure 1

Trends in ED visits annually, 2016-2019

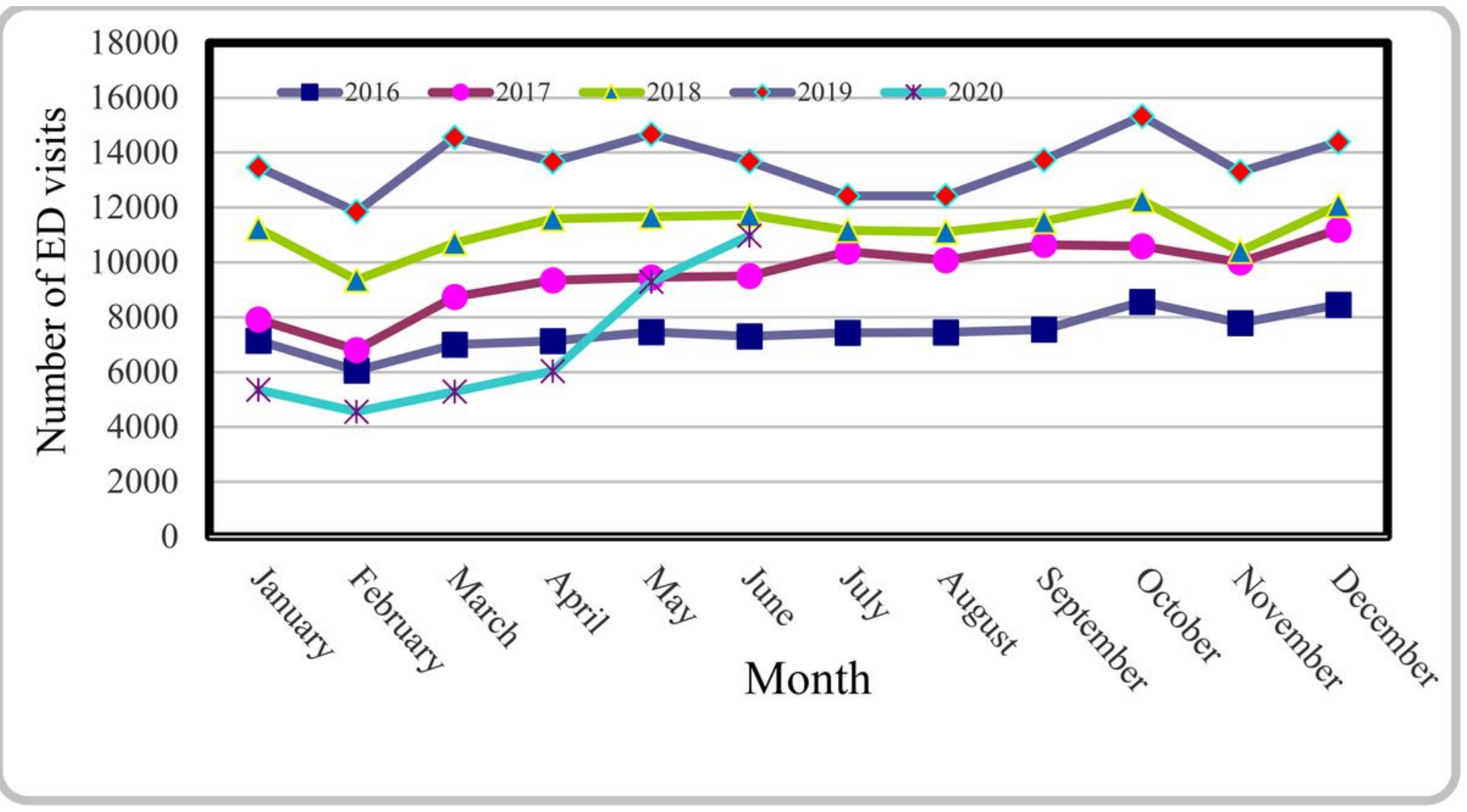

Figure 2 
Trends in ED visits monthly, 2016-2020

\section{Supplementary Files}

This is a list of supplementary files associated with this preprint. Click to download.

- Reference.rar 